\title{
Investigation of Fullerenes for High-Speed, Low Latency, Photonic Switching
}

H. W. H. Lee

R. N. Shelton

Febuary 13, 1997

This is an informal report intended primarily for internal or limited external distribution. The opinions and conclusions stated are those of the author and may or may not be those of the Laboratory.

Work performed under the auspices of the U.S. Department of Energy by the Lawrence Livermore National Laboratory under Contract W-7405-Eng-48. 


\section{DISCLAIMER}

This document was prepared as an account of work sponsored by an agency of the United States Government. Neither the United States Government nor the University of California nor any of their employees, makes any warranty, express or implied, or assumes any legal liability or responsibility for the accuracy, completeness, or usefulness of any information, apparatus, product, or process disclosed, or represents that its use would not infringe privately owned rights. Reference herein to any specific commercial product, process, or service by trade name, trademark, manufacturer, or otherwise, does not necessarily constitute or imply its endorsement, recommendation, or favoring by the United States Government or the University of California. The views and opinions of authors expressed herein do not necessarily state or reflect those of the United States Government or the University of California, and shall not be used for advertising or product endorsement purposes.

This report has been reproduced directly from the best available copy.

Available to DOE and DOE contractors from the Office of Scientific and Technical Information

P.O. Box 62, Oak Ridge, TN 37831

Prices available from (615) 576-8401, FTS 626-8401

Available to the public from the

National Technical Information Service

U.S. Department of Commerce 5285 Port Royal Rd.,

Springfield, VA 22161 


\title{
Final Report
}

\section{INVESTIGATION OF FULLERENES FOR HIGH-SPEED, LOW LATENCY, PHOTONIC SWITCHING}

\author{
96-LW-090 \\ Howard W. H. Lee, P.I. \\ Physics and Space Technology \\ Lawrence Livermore National Laboratory \\ Robert N. Shelton, co-P.I. \\ Department of Physics \\ University of California at Davis
}

\begin{abstract}
The components in high-speed, all-optical photonic systems must satisfy two essential requirements: (1) high swiching speeds in the range of Tbit/s, and (2) low latency, where the latency is the amount of time that the optical signal remains in the device. An important problem precluding the practical implementation of high-speed, all-optical switching is the lack of a material with appropriate nonlinear optical properties needed to effect the switching. Numerous material systems have been studied in the past, but none have met all the necessary requirements. Development of such a material and its incorporation into photonic devices would advance the field tremendously. This Lab-wide LDRD project resolved this critical problem.
\end{abstract}


We investigated the nonlinear optical properties of fullerenes and fullerene derivatives and demonstrated all-optical switching using their thin films. This research focused on their potentially large and fast nonresonant third order optical nonlinearity. Our studies showed that, unlike other promising nonlinear optical materials, the figure-of-merit of fullerenes for photonic switching exceeds the acceptable level. This differentiates fullerenes from other promising material systems.

\section{Background}

All-optical networks are emerging as the next generation of technology for high-speed telecommunications and computing. Problems arise in the limitations imposed by present-day electronics on the projected requirements for switching, routing, and signal-processing. Projections on the future requirements of high-speed optical networks indicate the need for optical switching and signal processing devices that operate at much higher speeds, approaching terabits per second (Tbit/s) and greater, than attainable with electronics or optoelectronics [1]. These speeds are needed to fully exploit the enormous capabilities of future fiber-optic networks. Larry Smarr, Director of the United States National Center for Supercomputing, recently stated that we are on the threshold of a "tera era" where telecommunications networks and switches must handle terabit/s data rates, and individual components in computer and communications systems must respond in sub-picosecond time scales. This technology will also prove to be critical to the implementation of emerging DOE Defense Program missions such as the Accelerated Strategic Computing Initiative (ASCI), where high-speed switching systems are required for the practical implementation of a "supercomputer." The only way currently known to achieve the projected speed requirements is with all-optical systems that incorporate photonic devices [1].

Advanced all-optical networks rely on novel devices that multiplex, route, and detect optical information. The strict requirements on these components include: (1) high-speeds in the range of Tbit/s, and (2) low latency, where latency is the amount of time the optical signal remains in the device. Clearly, high-speed switching demands low latency for synchronization purposes. An important problem precluding the practical implementation of high-speed optical switching is the 
lack of a material with appropriate nonlinear optical properties needed to effect the switching and that can be easily and economically incorporated into useful devices. Numerous material systems have been studied for this purpose, but none have met all the necessary requirements [2]. Development and incorporation of such a material into photonic devices would advance the field tremendously. This project addressed and solved this critical problem by implementing fullerenes (specifically $\mathrm{C}_{70}$ ) as the nonlinear optical material.

All-optical or photonic switching involves the control of an optical beam or pulse with another optical beam or pulse and relies on at least one of the following light-matter interactions:

(1) Optically-induced changes in the relative optical phase between two beams, which changes their optical interference. Switching is effected within an interferometer.

(2) Optically-induced power exchange between two optical beams

(3) Optically-induced polarization changes

(4) Frequency changes in a temporal soliton pulse due to interaction with another copropagating, orthogonally polarized soliton

(5) Changes in arrival time and phase shift in a soliton pulse due to a collision with another soliton beam.

The key material property is an intensity-dependent refractive index $n(I)=n_{0}+n_{2} I$ that allows light to control light. Here, $\mathrm{n}_{0}$ is the "normal" or linear refractive index, and $\mathrm{n}_{2}$ is the nonlinear refractive index (Kerr index) that is related to the real part of the complex third order optical nonlinearity.

Currently, most photonic switching is envisioned using optical fibers because they have one of the largest figure-of-merit (FOM) for all-optical switching presently known. A FOM for photonic switching can be defined as $\mathrm{FOM} \equiv 2 \mathrm{n}_{2} / \beta \lambda>1$, where $\mathrm{n}_{2}$ is the nonlinear index of refraction and $\beta$ is the two-photon absorption coefficient. This definition of the FOM assumes that switching requires a $\pi$ phase shift. It balances the benefits of a large $n_{2}$ with the detriment of a large $\beta$, and must exceed 1 for acceptable photonic switching performance. The large FOM for optical fibers results from small two-photon absorption in the glass comprising the fiber. The 
nonlinear index $\left(\mathrm{n}_{2}\right)$ is actually quite small. Consequently, all-optical fiber switches require extremely long lengths of fiber (100's of meters to kilometers) to accumulate the desired phase shift needed to effect efficient switching. Size and latency become critical limiting issues with a fiber optics approach. Clearly, optical fibers will not work and another material system is needed. Thus, the problem for efficient optical switching can be summarized as a search for appropriate nonlinear optical materials that satisfy the FOM requirements (at the important wavelength range for optical communications or optical interconnectsi.e., $800-900 \mathrm{~nm}$ and $1.3-1.5 \mu \mathrm{m}$ ), with the caveat that $\mathrm{n}_{2}$ be large enough so that high control pulse powers and/or long interaction lengths are not required. This allows full integration of the optical switch onto a chip. Furthermore, the response time of the nonlinearity and the configuration of the device must allow operation in the Tbit/s regime.

We investigated the nonlinear optical properties of fullerenes and fullerene derivatives for all-optical photonic switching applications using an integrated optics approach. Fullerenes (such as $\mathrm{C}_{60}$ and $\mathrm{C}_{70}$ ) are a new class of recently discovered carbon molecules with attractive properties for photonic applications. This research focused on their potentially large and fast nonresonant third order optical nonlinearity for broadband photonic switching applications. Fullerenes have the potential for extraordinarily large optical nonlinearities due to the presence of large polarizable electron densities, though the precise origin and nature of the optical nonlinearity are not wellunderstood. Appropriate chemical modification of the basic fullerene chemical structure can enhance the nonlinearity. Incorporation of a metal atom, such as a rare earth atom, inside the fullerene cage results in an endohedral metallofullerene with a highly polarizable electron density that is predicted to have nonlinearities an order of magnitude or more greater than the empty fullerene counterpart. The basic structure of fullerenes and its modification result in electronic structures that lead to potentially enormous nonlinear optical properties. Our results (to be discussed below) show that fullerenes are a unique material system. Unlike many other promising nonlinear optical materials (e.g., GaAs, organic polymers, etc.), the FOM of fullerenes for 
photonic switching exceeds the acceptable level. This clearly differentiates fullerenes from other promising material systems.

\section{Goals and Proposed Work}

Our goals were to develop a fundamental understanding of the nonlinear optical properties of fullerenes and fullerene derivatives, and apply that towards high-speed (Tbit/s), low latency (spicoseconds), integrated, all-optical switching. This effort is highly leveraged on physics, chemical synthesis, characterization, and fabrication capabilities already available at LLNL and at the University of California at Davis.

This project involved: (1) fundamental scientific studies of the nonlinear optical properties of various doped, undoped, and chemically modified fullerene thin films, (2) synthesis of various fullerene derivatives guided by the knowledge derived from our studies on the origin of the optical nonlinearity, (3) demonstration of photonic switching with these fullerene systems, and (4) development of fullerene-based nonlinear optical waveguides for integrated optical switching devices.

\section{Results and Discussion}

We investigated the nonlinear optical properties of various doped and undoped fullerenes, and chemically modified fullerenes to determine their effectiveness for photonic switching. $\mathrm{C}_{60}$ has been studied by numerous researchers and has been found to lack the appropriate nonlinear optical properties. We focused on the less symmetrical, higher order fullerenes ( $\mathrm{C}_{70}$ and above), where the proper combination of geometric asymmetry, electronic structure, linear absorption, and electron-phonon coupling may give rise to the proper nonlinear optical properties. Endohedral metallofullerenes are also particularly attractive. The nonlinear optical properties of endohedral metallofullerenes arise from charge transfer within the molecule. During formation of the metallofullerene, a charge transfer takes place between the metal atom and fullerene, forming a metal cation and corresponding fullerene anion. The metal atom inside the shell gives up electrons 
that are then transferred to the fullerene molecule and dispersed over the fullerene anion in a diffuse, highly polarizable orbital. This highly polarizable orbital gives the fullerene anion a predicted nonlinearity an order of magnitude or more larger than its empty counterpart. However, time constraints prevented a study into endohedral metallofullerenes, and will be reserved for future investigations.

Fullerene samples were obtained both commercially and through chemical synthesis. $\mathrm{C}_{70}$ and a few higher order fullerenes are now commercially available. However, doped and chemically modified fullerenes, and metallofullerenes are not readily available and must be synthesized. Professor Robert Shelton and his group in the physics department at U. C. Davis have expertise and experience in the fabrication and chemical modification of various fullerenes, along with their comprehensive characterization and analysis capabilities (e.g., X-ray diffraction, Differential Thermal Analysis, resistivity, magneticism) [3]. This ensured the availability of new and well-characterized material.

Evaluation of the optical nonlinearity of these fullerene systems was a critical task. A sensitive and accurate method for measuring nonlinear optical properties was needed. Many techniques are available (e.g., Z-scan and degenerate four-wave mixing), but the large variance in the reported values of nonlinear optical constants (some differ by several orders of magnitude) reveals the inadequacies of these techniques. To circumvent this problem, we used a new technique we invented at LLNL that is one of the most simple, sensitive, and accurate techniques for nonlinear optical measurements. This technique, which we named Antiresonant Ring Interferometric Nonlinear Spectroscopy (ARINS) [4], accurately measures the complex nonlinear optical properties of a material. 


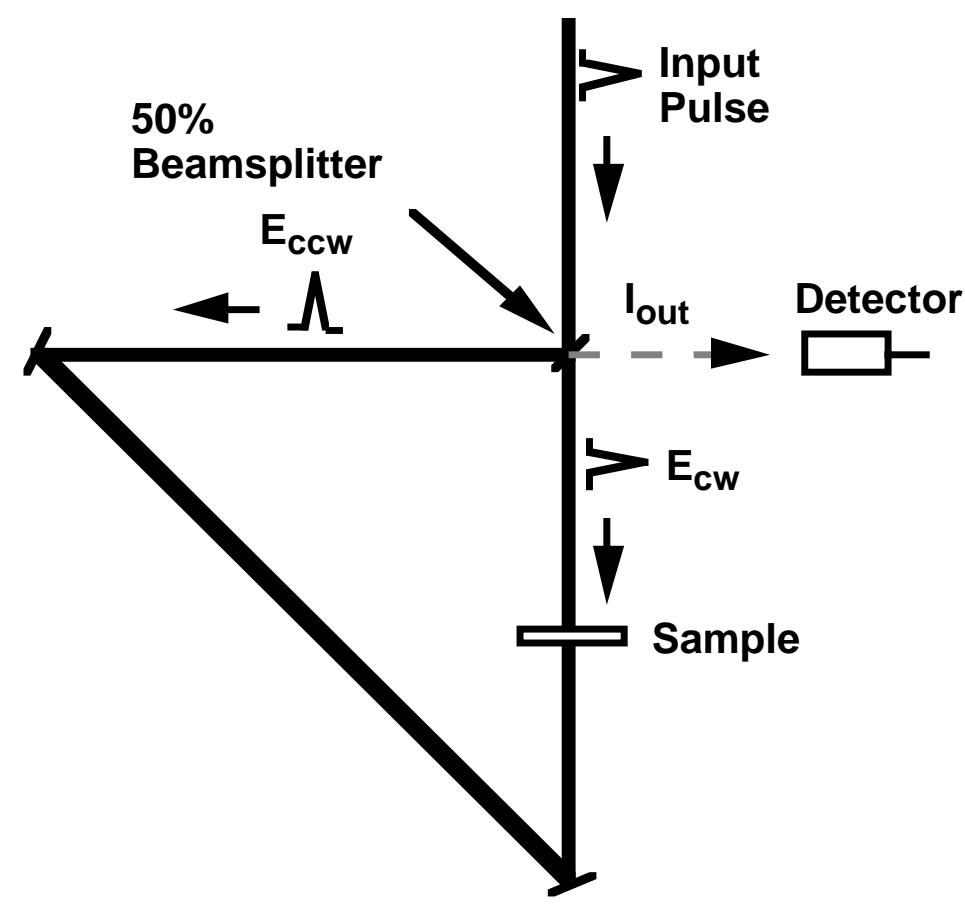

Figure 1. ARINS technique for nonlinear optical measurements [4].

Briefly, the ARINS technique (shown in Figure 1) works in the following manner. A nominally $50 \%$ beamsplitter divides an input pulse into two counterpropagating pulses. The sample is positioned in the antiresonant ring (ARR) interferometer to prevent the temporal overlap of the two pulses at the sample. After traversing the ARR with precisely the same physical path, both pulses interfere at the beamsplitter. The ARR output, $I_{\text {out }}$, is given by $I_{\text {out }} \propto\left|E_{c w}-E_{c c w}\right|^{2}$, where $E_{c w}$ and $E_{c c w}$ are the optical fields after traveling clockwise and counter-clockwise around the ring, respectively. If exactly $50 \%$ beamsplitting is used and if both pulses encounters the same interactions, then the ARR is said to be balanced. This results in zero output with all the optical energy returning to the input. The key to ARINS is configuring the ARR such that the two counterpropagating pulses initiate nonlinear responses of different magnitudes. Ideally, only one of the two pulses initiates the desired nonlinear response. This unbalances the ring and results in output from the ARR that is directly related to the nonlinear properties of the material. Further, 
$I_{\text {out }}$ appears against a dark background, which greatly improves its sensitivity. We have already demonstrated the sensitivity and accuracy of this technique in various material systems [4].

Our nonlinear optical measurements on $\mathrm{C}_{70}$ thin films using ARINS revealed large Kerr indices $\left(\mathrm{n}_{2}\right)$, moderate two-photon absorption coefficients $(\beta)$, and fast nonlinear optical response times with a FOM that is competitive with optical fibers. Our ARINS measurements covered the spectral range from $710-900 \mathrm{~nm}$ [5]. We found large $\mathrm{n}_{2}$ 's ranging from $2.8 \times 10^{-11}$ to $1.5 \times 10^{-9}$ $\mathrm{cm}^{2} / \mathrm{W}$ and moderate two-photon absorption coefficients ranging from $1.9 \times 10^{-8}$ to $3.4 \times 10^{-5}$ $\mathrm{cm} / \mathrm{W}$. This represents the first measurements of this kind and over such a broad spectral range.

We also investigated the ground and excited state electronic properties of these fullerenes with various general and time-resolved spectroscopic techniques (e.g., transient absorption and photoluminescence, Kerr gate, pump-probe, transient grating, photon echoes). This provided an essential understanding of the electronic structure and dynamical behavior of fullerenes, which is critical to developing a thorough understanding of the origin and nature of the optical nonlinearity. We found that the response time of the resonant and nonresonant optical nonlinearity range from picoseconds (ps) to femtoseconds (fs). Figure 2 shows a Kerr gate experiment on a $160 \mathrm{~nm}$ thick $\mathrm{C}_{70}$ thin film using $60 \mathrm{fs}$ optical pulses. The result is a pulsewidth-limited response, indicating that the third-order optical nonlinearity can be switched faster than $60 \mathrm{fs}$. This translates to potentially $\geq 15 \mathrm{THz}$ bandwidth for the temporal response of the third order optical nonlinearity of $\mathrm{C}_{70}$ thin films. These measurements show that high speed (Tbit/s) switching is possible with $\mathrm{C}_{70}$ thin films.

Our results predict that the much larger $\mathrm{n}_{2}$ along with the ultrafast nonlinear optical response of $\mathrm{C}_{70}$ enable considerably smaller switches (millimeters) in an integrated optics approach and potentially high-speed operation in the Tbit/s regime. This effectively solves the critical latency problem and enables high speed-operation.

We have also demonstrated the first fullerene-based all-optical switches. The basic switch configuration consisted of a $\mathrm{C}_{70}$ thin film in an antiresonant ring (ARR) interferometer. Long 
lengths of optical fibers were not required. We used the same configuration as in the ARINS nonlinear measurements. The output from the switch is shown in Figure 3. For demonstration

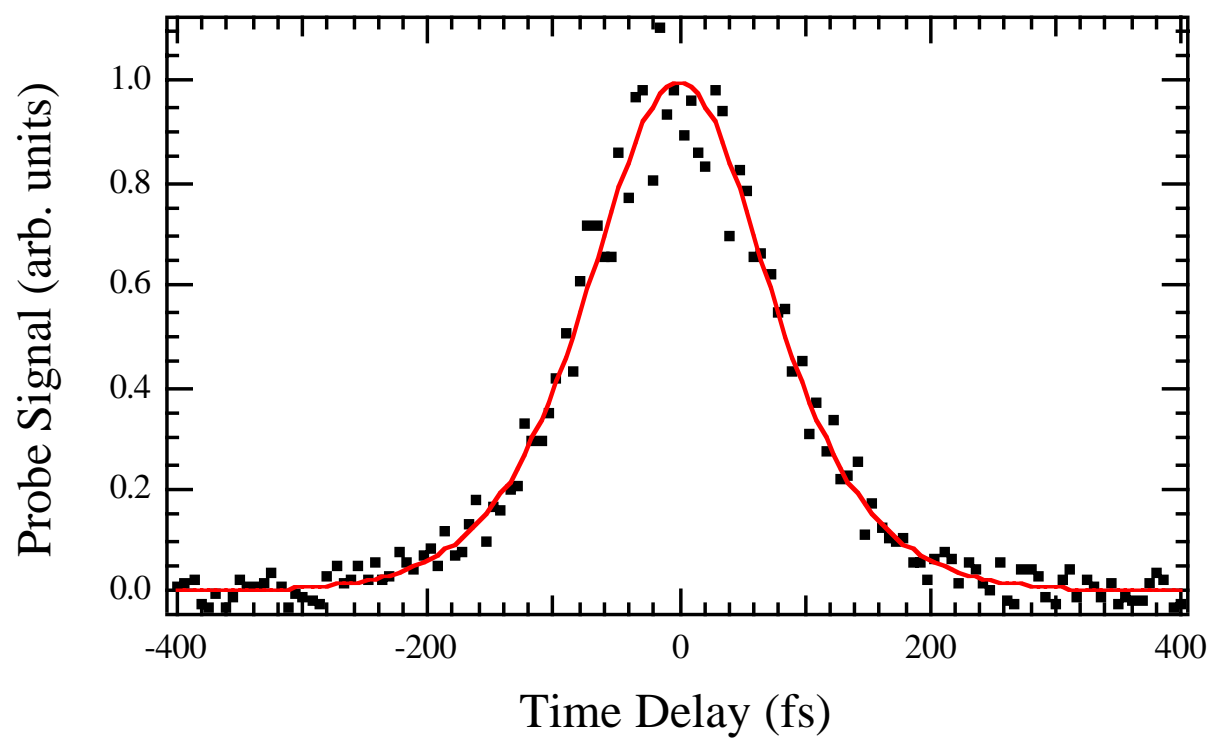

Figure 2. Kerr gate experiment showing ultrafast temporal response of third-order optical nonlinearity of $\mathrm{C}_{70}$ thin film.

purposes, a laser beam at $840 \mathrm{~nm}$ served as the input to the switch. The control pulses, which effect the switching, are derived from a femtosecond laser operating at $840 \mathrm{~nm}$ and at an $82 \mathrm{MHz}$ repetition rate. In Figure 3, the output pulses at $82 \mathrm{MHz}$ clearly verify the all-optical switching operation. We have also shown that the switch can function as an all-optical demultiplexor for applications in time division multiplexing (TDM), and as an all-optical AND logic gate. These operations are shown in figures 4 and 5. In this initial effort, we used a conservative switching speed of $82 \mathrm{MHz}$ along with a switching energy of a few picojoules. This is a preliminary result and operating parameters such as switching speed and energy have not been optimized. However, if the femtosecond dynamics of the nonlinear optical response can be directly translated into switching speed, then the anticipated Tbit/s switching rates will be realized. 


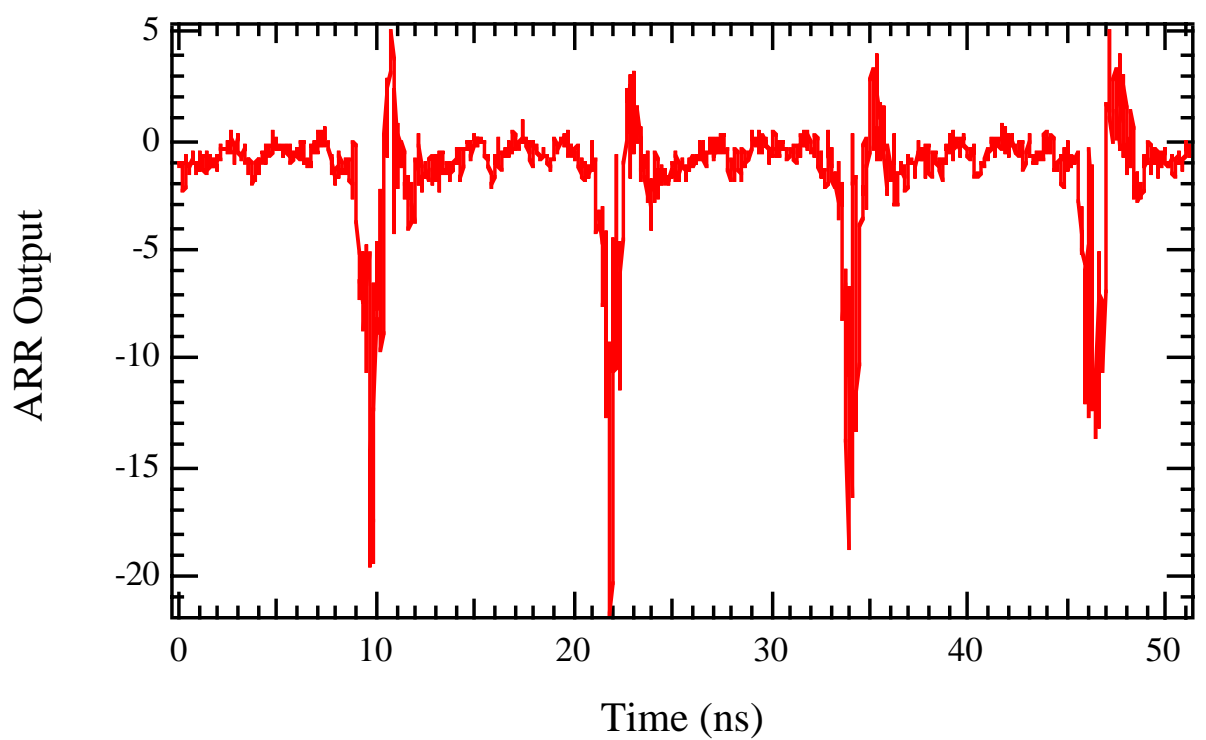

Figure 3. Switched output from an all-optical switch made from a $160 \mathrm{~nm}$ thick $\mathrm{C} 70$ thin film.

Input

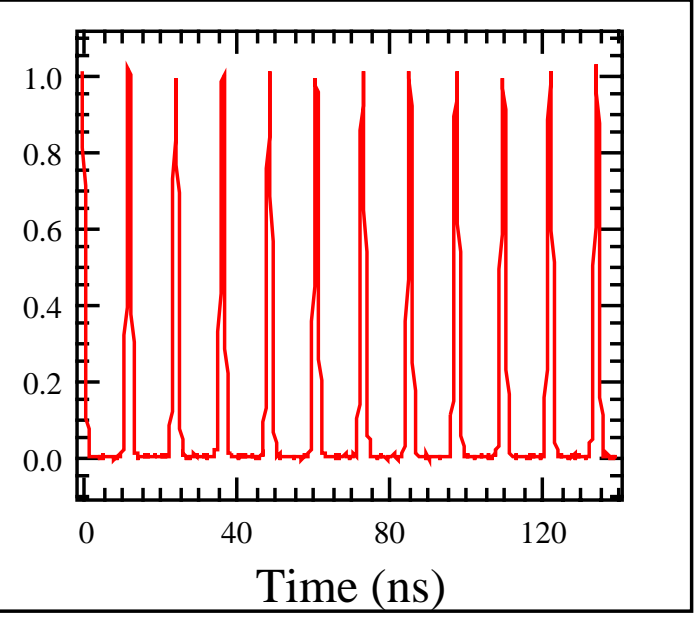

Switched Output

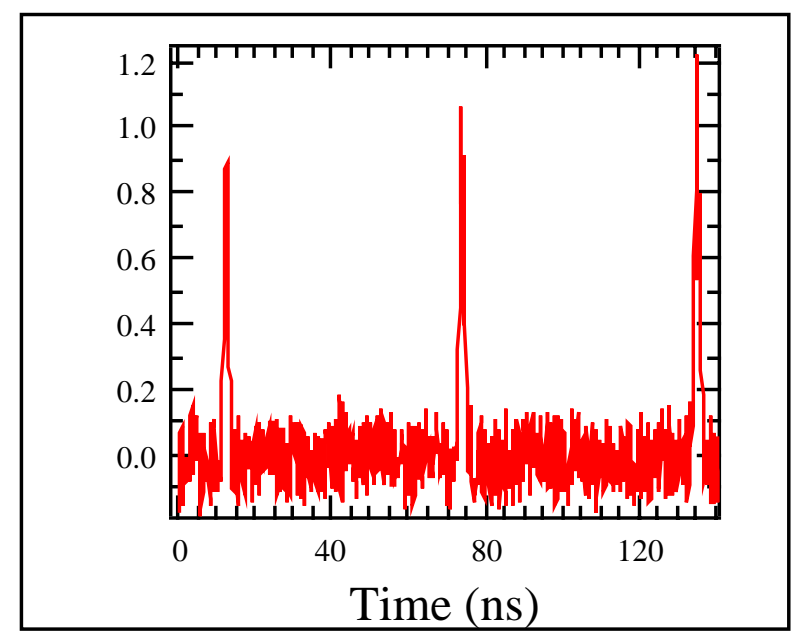

Figure 4. Demonstration of all-optical demultiplexing using $\mathrm{C}_{70}$ thin film in an ARR. Every fifth input pulse is switched out to simulate demultiplexing 
Input A
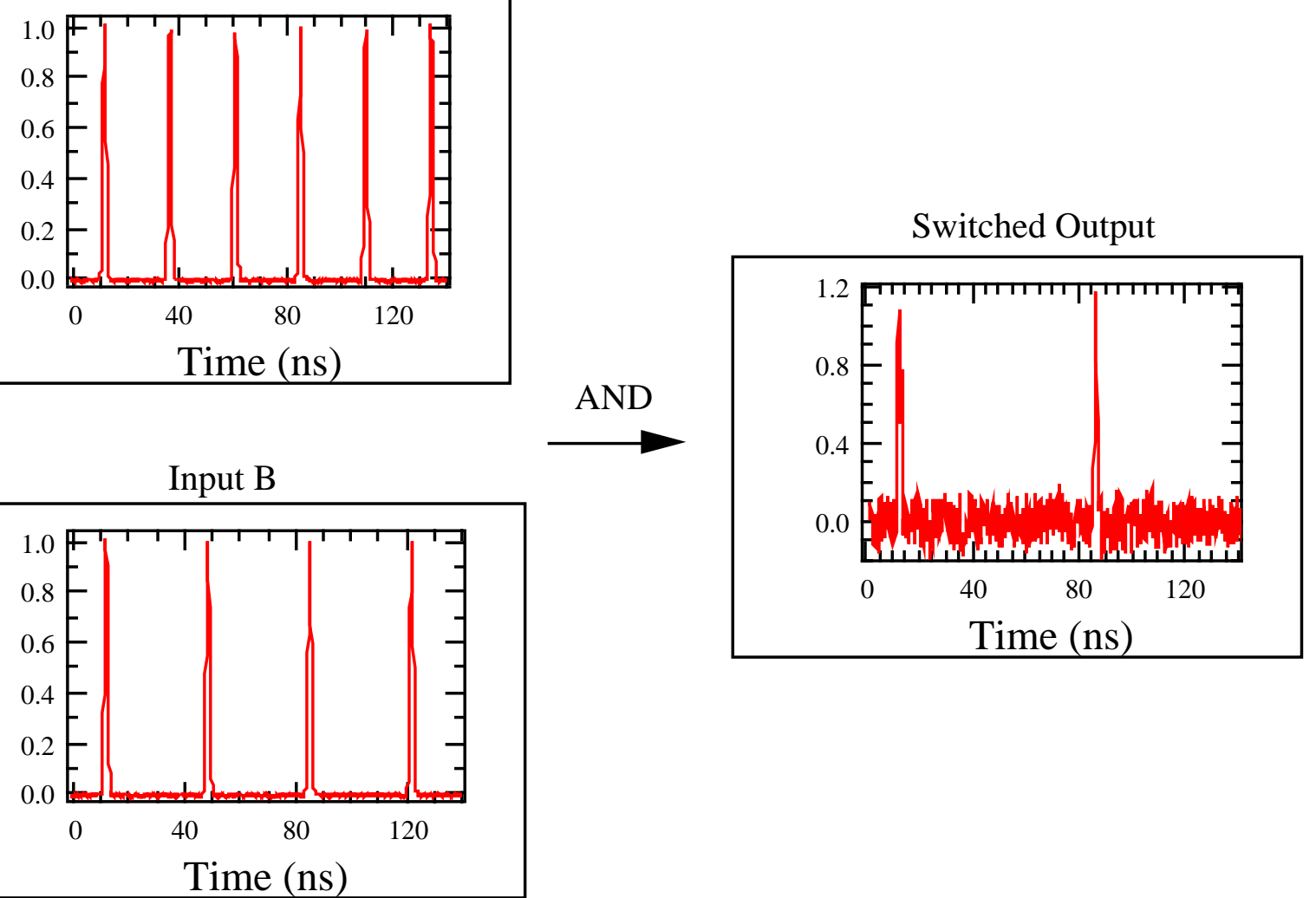

Figure 5. Demonstration of all-optical AND logic gate using $C_{70}$ thin film in an ARR. The switched output represents the reult of an AND operation on Input A and Input B.

To implement the integrated optics approach we proposed, optical waveguides needed to be fabricated from fullerenes. We demonstrated the patterning of $\mathrm{C}_{60}$ thin films into channels for guided wave integrated optics [6]. This was achieved by vapor deposition of $\mathrm{C}_{60}$ onto etched polymer photoresist that resided on a silicon wafer covered with a thin layer of silicon oxide. The guided wave structure was then defined by solvent liftoff of the polymer photoresist. Though the structures proved to be too small to effectively couple significant light through them, atomic force microscopy (AFM) showed that the side and top walls of the waveguide were extremely smooth, a necessary condition for lossless light propagation. Thus, wave guiding was only limited by our inability to effectively couple light into the waveguide. In future studies, we will continue to study this process and explore other techniques for fabricating nonlinear waveguides from fullerenes. 
Another particularly promising method involves incorporating fullerenes into various matrices such as polymers, glasses, and sol-gels. These matrices impart an enormous versatility to this material system and offer extremely interesting host matrices to study the electronic, physical, and optical properties of fullerenes. They allow easy processing and results in simple and convenient systems for shaping waveguides into any desired geometry. Our integrated optics approach will enable considerably smaller devices and effectively eliminate the critical latency problem.

\section{Summary}

What differentiates fullerenes from other nonlinear optical material systems that have been proposed for implementing high-speed, low latency photonic switching is that they work. We

have measured and evaluated the third order nonlinear optical properties of $\mathrm{C}_{70}$ thin films and demonstrated that they can be used to effect all-optical switching. Though fullerenes are a new class of materials, their unique optical and nonlinear properties warrant close scrutiny for photonic applications. Our work demonstrated that extremely high-speed, low latency photonic switching may be uniquely attainable with fullerenes as the nonlinear optical material. This eliminates one of the most important limiting issue for future high-speed networks and computers. 


\section{References}

[1] P. W. E. Smith, “All-Optical Devices: Materials Requirements," in Nonlinear Optical Properties of Advanced Materials, SPIE Proceedings, Vol. 1852, S. Etemad, Ed., 1993.

[2] G. I. Stegeman and A. Miller, "Physics of All-Optical Switching Devices," in Photonics in Switching, J. Midwinter, Ed., Academic Press, Cambridge, MA, 1993.

[3] (a) T. T. Anderson, P. L. Dyer, J. W. Dykes, P. Klavins, P. E. Anderson, J. Z. Liu, and R. N. Shelton, "A Plasma Arc Reactor for Fullerene Production," Rev Sci Instr, 65, 3820(1994).

(b) J. W. Dykes, P. Klavins, M. D. Lan, J. Z. Liu, and R. N. Shelton, "Production of SingleCrystal K3C60," J. of Superconductivity, 7, 635(1994).

(c) J. Z. Liu, J. W. Dykes, M. D. Lan, P. Klavins, R. N. Shelton, and M. M. Olmstead, “Vapor Transport Growth of C60 Crystals," Appl. Phys. Lett., 62, 531(1993).

[4] H. W. H. Lee and R. S. Hughes, Jr., "Antiresonant Ring Interferometric Nonlinear Spectroscopy for Nonlinear Optical Measurements," Optics Letters, 19, 1708(1994)

[5] (a) H. W. H. Lee, J. Diane Cooke, and J. E. Davis, "Fullerene Thin Films for High-Speed All-Optical Switching," Fullerenes and Photonics II, SPIE Proceedings Vol. 2530, 1995;

(b) H. W. H. Lee, "Investigation of Fullerenes for Optoelectronic and Photonic Applications," 26th Boulder Damage Symposium on Optical Materials for High Power Lasers, Boulder, CO, October 24-26, 1994, invited talk; 
(c) H. W. H. Lee, R. S. Hughes, Jr., J. E. Davis, C. F. McConaghy, A. V. Hamza, and M. Balooch, "Feasibility of Fullerene Thin Films for High-Speed All-Optical Switching," Conference on Lasers and Electro-Optics, Anaheim, CA. May 8-13, 1994.

[6] A. V. Hamza, M. Balooch, R. J. Tench, M. A. Schildbach, H. W. H. Lee, and C. McConaghy, "Patterning of C60 Films," J. Vac. Sci. \& Technol. B, 11, 763(1993). 


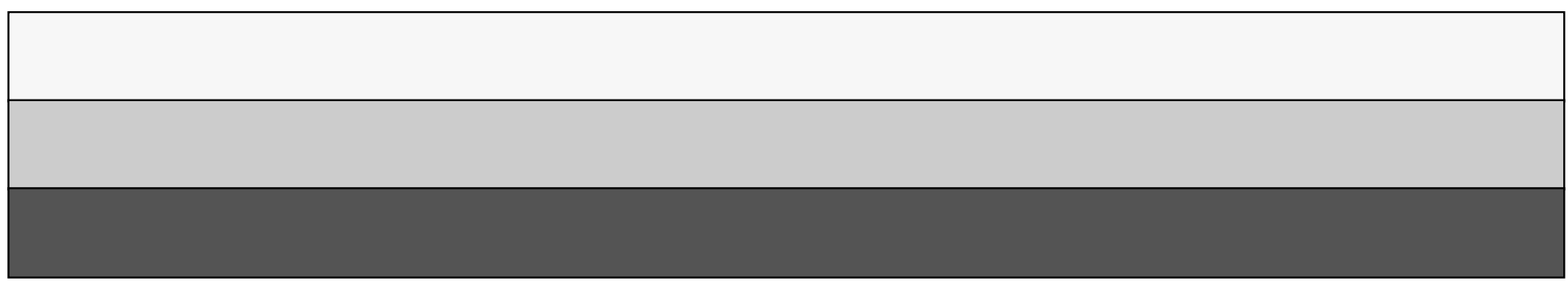

